\section{Donación y trasplante de órganos: propuesta desde la Academia Chilena de Medicina}

\author{
JUAN HEPP ${ }^{1}$, JUAN PABLO BECA ${ }^{1}$, \\ SERGIO MORAN ${ }^{1}$, EMILIO ROESSLER ${ }^{1}$, \\ MARIO URIBE ${ }^{1}$, JOSÉ MANUEL PALACIOS ${ }^{2}$
}

\section{A proposal of the Chilean Academy of Medicine to improve organ procurement and transplantation in Chile}

The Chilean Academy of Medicine designated a group of specialists to evaluate the practice and to propose reforms for organ donation and transplantation, due to the general insufficiencies at the national level with these procedures. In the last six years the mean number of organ transplants in Chile was 340 cases per year while effective cadaveric donors ranged between 6 and 10 per million inhabitants. These averages remained stable during this period and are among the lowest in the region. Our analysis attributed these deficient results mainly to low organ donation and inefficient procurement due to lack of compliance with protocols and little accountability. The committee proposes several measures for improvement. These are a systematic and obligatory report of potential organ donors by all emergency and critical care centers, frequent evaluation of results, empowering of health authorities to correct insufficiencies in organ procurement, education programs for primary, secondary, technical and university students to improve their knowledge about the social significance and solidarity required for transplantation policies and specialized updated training of all health professionals involved. Organ donation and transplantation must be based on clear and fair ethical considerations in order to be accepted by the general public.

(Rev Med Chile 2020; 148: 381-386)

Key words: Organ Transplantation; Public Policy; Tissue and Organ Procurement

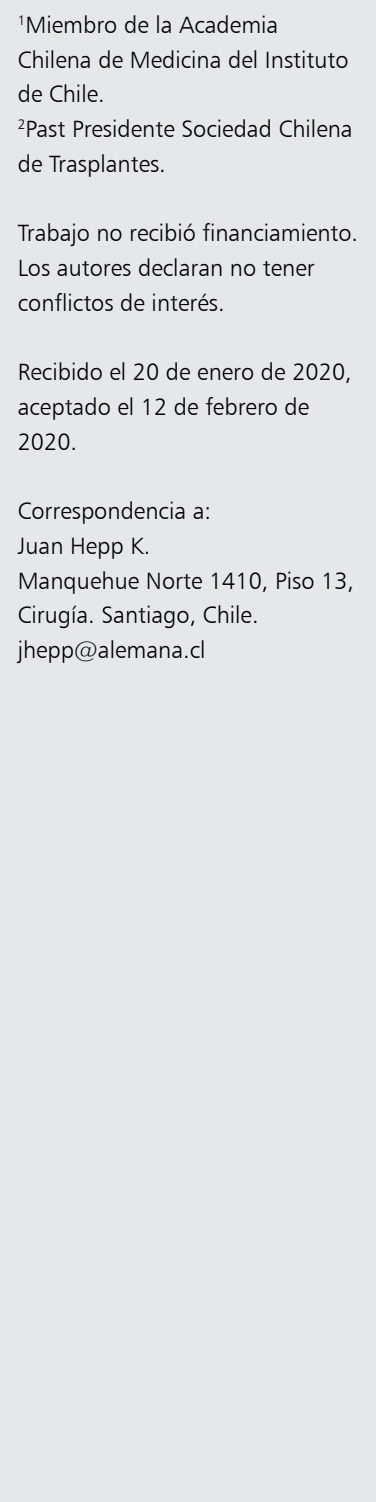

Chilena de Medicina del Instituto de Chile.

2Past Presidente Sociedad Chilena

Trabajo no recibió financiamiento. Los autores declaran no tener

Recibido el 20 de enero de 2020 aceptado el 12 de febrero de

Correspondencia a:

Juan Hepp K

Ciso 13 Cirugía. Santiago, Chile

jhepp@alemana.cl

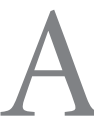
nte la importancia para la salud pública de los trasplantes de órganos y la realidad nacional, que muestra insuficiencias en la materia, la Academia Chilena de Medicina decidió formar un comité para evaluar la situación actual y proponer mejoras a las autoridades de salud y a la comunidad nacional.

En Chile, los trasplantes de órganos sólidos (TXO) se inician en la década de 1960-69, poco tiempo después que estos se realizaran a nivel mundial. Hasta la fecha, se han efectuado en nuestro país más de once mil TXO. Los resultados obtenidos en términos de supervivencia de pacientes, de órganos y complicaciones del procedimiento, son similares a las de los mejores centros internacionales. Durante los últimos 6 años, el promedio anual de TXO con órganos de cadáver en nuestro país ha sido de $340^{1,2}$.

A pesar de los buenos resultados clínicos, los datos actuales muestran que el número de tras- 
plantes se ha mantenido sin variaciones durante los últimos 6 años. Esto es debido, en gran parte, a escasez de donación de órganos. La afirmación "sin donantes no hay trasplantes" es indiscutible. Por este motivo el comité decidió poner énfasis en el proceso de donación de órganos. Estos donantes son principalmente pacientes con daño cerebral catastrófico por traumatismo encefalocraneano o accidente cerebrovascular, que evolucionan a muerte encefálica.

Las tasas de donación a nivel mundial fluctúan entre 2 y 47 por millón de habitantes (PMHA). En Europa la tasa promedio es 22, destacando España con 47 donantes PMHA. En Estados Unidos de Norteamérica (EE. UU.) la tasa es 32. En Hispanoamérica, países como Uruguay, Argentina y algunos estados en Brasil, tienen tasas de donación por sobre $20 \mathrm{PMHA}^{3-5}$. En Chile, en los últimos 10 años, nuestra tasa ha oscilado entre 6 y 10 PMHA, siendo de las más bajas en la región. Además, esto se ve agravado porque la necesidad de órganos ha aumentado en las últimas décadas, debido a los mejores resultados de corto y largo plazo con este tratamiento e indicaciones de trasplante en patologías nuevas y más complejas. Esto hace imperativo aumentar la tasa de donación.

Si tuviésemos, por ejemplo, la tasa de donación de España, podríamos lograr anualmente más de 800 donantes efectivos. Sin embargo, en los últimos 6 años hemos tenido entre 103 y 173 donantes anuales. Esto es consecuencia de una insuficiente detección, fallas en la interacción entre médicos tratantes y coordinadores de trasplantes, sumado a una alta tasa de rechazo familiar. Así, el número de trasplantes que se realizan en nuestro país es muy insuficiente para las necesidades de la población. Para alcanzar los mejores estándares internacionales, los donantes potenciales deben ser detectados oportunamente, mantenidos en una unidad de pacientes críticos (UPC), luego obtener el consentimiento de donación y, finalmente, coordinar con los equipos de trasplantes la procura de los órganos que corresponda. Si logramos cumplir con eficiencia estas etapas del proceso, nuestros resultados mejorarían significativamente $e^{6-9}$.

Actualmente alrededor de 80 a $85 \%$ de los donantes cadavéricos son originados en el sistema público y 15 a $20 \%$ en instituciones privadas, que se coordinan y complementan eficientemente. La mayoría de los trasplantes se realizan en la Región Metropolitana. A nivel nacional, entre los años 2010 y 2017, hubo 863 donantes cadavéricos efectivos en el sistema público y 146 en el sistema privado. Si se mide la capacidad de lograr donantes efectivos por centro, se observa una gran variabilidad, aunque cuenten con una estructura, número de camas, recursos humanos y población asignada semejante. La cifra de donantes cadavéricos efectivos procurados por centro para estos años, osciló entre 1 y 21 por centro. Necesitamos identificar los factores que generan estas diferencias para proponer las soluciones respectivas ${ }^{10}$.

El año 2010, el Ministerio de Salud (MINSAL) asumió la coordinación nacional de trasplantes, con la responsabilidad de organizar el procuramiento y los trasplantes de órganos ${ }^{11}$. Esta red de coordinadores se distribuye en instituciones públicas a lo largo del territorio nacional, donde existe infraestructura suficiente para la pesquisa, mantención de potenciales donantes y la realización de trasplantes. A nivel privado se generó un sistema similar que también es parte de la coordinadora nacional ${ }^{12}$. De esta forma, se constituyó un modelo semejante a los que funcionan en países con buenos resultados en donación de órganos y trasplantes, como son España, otros países de la Comunidad Europea y EE. UU. ${ }^{13}$.

Las leyes y reglamentos sobre donación y trasplantes de órganos y tejidos son consideradas pilares en el progreso de esta actividad. Es responsabilidad del Estado elaborarlas y vigilar su cumplimiento, como se expresa en la Declaración de Estambul $2008^{14}$. Nuestra historia de cuerpos legales relacionados con los trasplantes y la donación de órganos se inicia el año 1963 (Ley 15.262). Bajo este marco se realizaron los primeros TXO con donantes vivos y de cadáver.

La ley 18.173 del año 1982 introdujo los conceptos de donante vivo, de cadáver y la muerte encefálica (ME), lo cual se expresa en el reglamento solo para efectos de trasplante. En 1994, durante el trámite parlamentario de la ley 19.451, el concepto de ME fue impugnado ante el Tribunal Constitucional, el cual, mediante un fallo favorable determinó que "la abolición total e irreversible de todas las funciones encefálicas constituye la muerte real, definitiva, unívoca e inequívoca del ser humano". Esta ley y su reglamento definieron adecuadamente el proceso de donación de órganos y trasplantes, el concepto de ME y su diagnóstico, entre otros aspectos.

Posteriormente se publicaron varias leyes, no 
siempre en sintonía con los expertos en trasplantes. En 2010 (ley 20.413) se definió quiénes son donantes y cómo manifiestan su voluntad, creando el Registro de Donantes en el Registro Civil. La ley 20.673 (año 2013) estableció el donante universal, salvo oposición en vida, expresado en una notaría y también en el Registro Civil al obtener cédula de identidad o carné de conducir. Una falla en la forma de llevar a cabo el registro generó que 4 millones de ciudadanos aparecieran como no donantes. En el año 2017 (ley 20.988) se permitió la donación cruzada entre vivos. Finalmente, la ley 21.145 (año 2019) corrigió los efectos adversos de la ley 20.413 y especifica que la voluntad de no ser donante debe ser ratificada en plazo de 12 meses, normalizando el registro de donantes. También obliga la notificación de un potencial donante. En 2018, el MINSAL estableció un compromiso de gestión en el número de donantes anuales por hospital.

La educación de la población chilena sobre la importancia de la donación de órganos no ha sido eficaz. Existe una alta tasa de rechazo familiar a la donación. Se han realizado campañas mediáticas a favor de la donación, pero su impacto ha sido poco duradero en el tiempo. No existe a nivel nacional un programa de educación sistemático, tanto a nivel escolar como de educación superior técnica y universitaria.

Hoy existen expertos y hay recursos destinados a esta actividad, sin embargo, no se ha logrado aumentar el número de donantes. Se ha observado que la Coordinadora Nacional de Trasplantes del MINSAL tiene limitada autonomía en su quehacer, con escasa capacidad de fiscalización efectiva y de intervención para modificar deficiencias y corregir errores en los centros de salud.

Los cambios culturales que privilegian el bienestar individual por sobre el interés de la comunidad pueden afectar la solidaridad entre las personas y producir desconfianza en las instituciones. Se sabe que conductas prodonación provienen de experiencias positivas de terceros, mientras que la de no donación aumenta por falta de información ${ }^{15}$. Otros factores que también atentan contra la donación son derivados del desconocimiento de la postura prodonación de la Iglesia Católica, factores educacionales, culturales y generacionales. Los que no desean donar son en general personas mayores de 40 años, con menor nivel educacional, sin experiencia en donación o trasplantes y sin participación en actividades de servicio comunitario. Otros rechazan la intervención del cadáver, desconociendo el concepto de muerte encefálica y privilegian la opinión familiar sobre la voluntad individual. Los que están a favor de donar, en cambio, son generalmente menores de 40 años con actitudes opuestas a las anteriores. El fundamento de su posición es la solidaridad y la eventual reciprocidad ${ }^{16}$.

También hay déficit en la formación y educación de los equipos de salud. Un estudio, basado en entrevistas al personal de un servicio de urgencia (SU) y UPC, demostró un variado espectro de conocimientos sobre donación y trasplantes, desde una actitud a favor hasta desconocimiento y mitos que crean barreras para la donación ${ }^{17}$. Otro reporte mostró bajo nivel de capacitación en algunos coordinadores locales de trasplante y la opinión que esta actividad determina un impacto negativo en su calidad de vida ${ }^{18}$.

La educación es fundamental para lograr una cultura favorable a la donación que permita satisfacer las necesidades de trasplante de una población, por lo que es imprescindible involucrar a todos los actores sociales. Los elementos básicos para obtener una percepción social favorable a la donación son la información y la credibilidad, que permita establecer vínculos de confianza entre el público y el personal sanitario. Se debe considerar una educación comunitaria, escolar, universitaria, de las carreras de la salud, medios de comunicación y redes sociales. El objetivo es instaurar dicha temática a nivel familiar, iniciando este proceso en la educación básica, destacando la importancia de la donación desde el punto de vista social, reforzándolos en enseñanza media, sumando los conceptos de muerte cerebral, donación con donante vivo y cadáver y el impacto social del trasplante en la calidad de vida de los receptores, ahorro en costos de hospitalización y en reinserción laboral ${ }^{19}$.

Otro aspecto importante del proceso de donación es el compromiso de todos los participantes en la red local, empezando por el director de hospital, quien debe velar por el cumplimiento de los protocolos y facilitar la solución de los problemas locales, logrando así que la institución supere las tasas de procuramiento históricas. Además, debe haber un adecuado control por parte de la autoridad sanitaria, realizando visitas de auditoría e incluyendo los protocolos de donación para 
trasplantes en los procesos de acreditación de los SU y UPC ${ }^{20}$.

A estos conceptos se agrega lo planteado en un documento del año 2017 de la Organización Nacional de Trasplantes de España, redactado junto a la Sociedad Española de Medicina Intensiva. El documento destaca que la donación de órganos debe formar parte de los cuidados al final de vida en pacientes con daño cerebral catastrófico. Incorpora el proceso de la donación como un elemento sanitario en los cuidados al final de la vida, los cuales deberían ser parte fundamental en los procedimientos, cultura y ética de las UPC y SU²0.

Las propuestas que se describen a continuación tienen sólidos fundamentos éticos ${ }^{21,22}$. Los sistemas de donación y trasplantes de órganos deben responder equitativamente a las necesidades y derechos de los pacientes, con el fundamento ético del deber de justicia en la asignación de recursos y prestaciones. Para las decisiones caso a caso, el fundamento debe ser el respeto a los valores y preferencias de los pacientes, a sus expresiones previas de voluntad, junto con el apoyo a las decisiones familiares a través de la debida información y orientación. Se trata del respeto a la autonomía de los pacientes, incluyendo a sus redes familiares ${ }^{23}$. Todo lo anterior exige profesionales debidamente formados en lo técnico y en lo ético. Finalmente, la necesaria confianza pública es también un fundamento ético que depende de la información, educación y transparencia de los sistemas.

A modo de conclusión, para aumentar las tasas de donación y mejorar la eficiencia del sistema de procura, se proponen las siguientes medidas concretas:

1. Pesquisa sistemática de posibles donantes en SU y UPC, con información inmediata a los coordinadores locales. Este proceso por sí solo puede mejorar significativamente la donación.

2. Información oportuna y prudente a los familiares, junto al necesario apoyo en su duelo y para las decisiones que necesitan tomar.

3. Disponibilidad de UPC para potenciales donantes, con recursos y gestión eficiente en hospitales acreditados.

4. Los profesionales de las carreras de la salud deberían conocer la importancia de la donación de órganos, criterios de muerte encefálica, indicaciones y contraindicaciones de trasplante.
5. Campañas masivas, constantes, en medios de comunicación y redes sociales para sensibilizar a la población, derribando mitos y transmitiendo confianza sobre la seguridad y transparencia del proceso, destacando los buenos resultados.

6. Enseñar que la muerte encefálica es sinónimo de muerte efectiva y que dicho concepto está universalmente aceptado. Es fundamental clarificar los conceptos de solidaridad, reciprocidad y autonomía, y la importancia de la manifestación de la voluntad anticipada, comunicando a sus familiares la decisión de ser donante en caso de muerte encefálica.

7. Instruir sobre el marco legal vigente, destacando que el sistema chileno de distribución de órganos es objetivo, transparente y justo, en base a criterios preestablecidos que otorgan puntajes independientes de la condición social, previsional o económica de los pacientes.

8. Los profesionales de la salud, al asumir cargos en SU, UPC y pabellón, deberían ser capacitados en esta materia y contar con un programa de contención por el posible impacto de esta actividad en su vida personal.

9. Las autoridades de Salud deben cumplir un compromiso de gestión sobre la pesquisa y donación efectiva de órganos para trasplante en sus instituciones.

10. Solicitar a las autoridades del Ministerio de Educación, Ministerio de Salud, facultades de Medicina y sociedades científicas que desarrollen actividades formativas relacionadas con la donación de órganos y trasplantes.

11. Crear progresiva y planificadamente centros con la capacidad de realizar trasplantes en la medida que aumente la donación de órganos, con la debida regionalización, coordinación y competencia.

12. Incorporar herramientas de comunicación propias de nuestros tiempos, como aplicaciones digitales que permiten notificar al Coordinador Local de Procuramiento de un posible donante en su servicio.

Agradecimientos: El Comité de Trasplantes y Donación de Órganos de la Academia Chilena de Medicina agradece a los siguientes profesionales por sus aportes al trabajo realizado: Francisca González Cohens, Ingeniera Civil, Proyecto Kefuri, Web Intelligence Centre, Facultad de Ciencias Físicas y Matemáticas, Universidad de 
Chile. Fernando González Fuenzalida, Director del Departamento de Medicina Oriente, Facultad de Medicina, Universidad de Chile; Jefe de la Unidad de Nefrología, Diálisis y Trasplante Renal, Hospital del Salvador. Juan Eduardo Sánchez Valenzuela, Coordinador Nacional de Trasplantes, MINSAL, Médico Jefe UPC Hospital San Juan de Dios. José Luis Rojas Barraza, Ex Coordinador Nacional de Trasplantes, MINSAL. Ana María Arriagada Urzúa, Comité de Trasplantes Colegio Médico de Chile Departamento Medicina Hospital del Salvador, Ex Presidenta Corporación del Trasplante. Javier Domínguez Cruzat. Ex Presidente Corporación del Trasplante, Urólogo Pontificia Universidad Católica de Chile.

\section{Referencias}

1. Ministerio de Salud, "Yo dono vida - Estadísticas". [En línea]. Disponible en: https://yodonovida.minsal.cl/ estadisticas/numero-donantes.html [Accedido: 24 de noviembre de 2019].

2. Informe anual de procuramiento y trasplantes de órganos en Chile. Ministerio de Salud 2017. [En línea]. Disponible en: https://www.minsal.cl/wp-content/ uploads/2018/01/Datos-P\%C3\%A1gina-Enero-Diciembre-2017.pdf

3. Newsletter Transplant. International figures on donation and transplantation 2018. [En línea]. Disponible en: https://aetha.org/2019/09/16/newsletter-transplant-2019-ont/

4. Matezans R. International figures on organ donation and transplantation 2013. Newsletter Transplant: 19: 96, 2014.

5. Tuttlenewhall JE, Krishinan JM, Levy MF, Mc Bride V, Orlowski JP. Organ donation and utilization in the United Sates 1998-2007. Am J Transplant. 9 (4): 879893, 2009.

6. Matezans R, Domínguez GB, Coll E, Mohillo B, Marazuela A. How Spain reached 40 deceased organ donors per million population. Am J Transplant 2017; 17 (2): 1447-54.

7. Matezans R, Domínguez GB, Coll E, Mohillo B, Marazuela A, de la Rosa B. The 40 donors per million population plan: an action plan for improvement for organ donation and transplantation in Spain. Transplant Proc. 2019; 41: 3453-6.

8. Miller LD, Gardiner SK, Gubler KD. Emergency department referral for organ donation: more organ donors and more organ per donors. Am J Surg. 2014; 207: 72833.
9. Kutsogianis D, Asthana S, Townsed D, Gurmeat S, Karvellas CJ. The incidence of potential missed organ donors in intensive care unit and emergency rooms: a retrospective study. Intensive Care Med 2013; 39: 14529.

10. Good practice guidelines in the process of organ donation (2006. nov). http://www.ont.es/Paginas/Home.aspx

11. Decreto de ley de trasplantes 20.413: 15/01/2011: creación de coordinadora nacional de trasplantes. https:// www.leychile.cl

12. Donación de órganos: reportaje (10 de enero de 2019). [En línea]. Disponible en: https://www.eldinamo.cl/ reportajes/2019/01/10/transplantes-15-hospitales-publicos-no-han-aportado-ningun-donante-desde-2010/ [Accedido: 24 de noviembre de 2019].

13. Marzolais P, Durand P, Charboney E, Serri K, Legale AM, Bernard F, Albert M. The first two years of activity of a specialized organ procurement center: report of an innovative approach to improve organ donation. Am J Transplant 2017; 17 (2): 1613-9.

14. Uribe M. Declaración de Estambul en relación al tráfico de órganos y turismo en trasplante. (Traducción). Rev Méd Chile 2009; 137: 1117-21.

15. Bustamante M y Villareal P. Motivación a la donación de órganos en Chile. Panorama Socioeconómico 2008; 26 (36): 86-97.

16. Solar S, Ovalle A, Simian ME, Escobar J, Beca JP. Tres factores que influyen en la actitud de las personas ante la donación de órganos. Rev. Chilena de Cirugía. 2008; 30 (3): 262-7.

17. Maldonado L, Vera M, Repeto P, Bernales M. Creencias del personal del Servicio de Urgencia y de la Unidad de Cuidados Intensivos respecto de su rol en la detección de potenciales donantes: un estudio desde el modelo de la conducta planificada. Libro de resúmenes del VII Congreso Chileno de Trasplante 2019, página 72. [En línea]. Disponible en: http://congreso2019.sociedaddetrasplante.cl/assets/libro-resumen-congreso-trasplante-2019.pdf, p. 72.

18. Alcayaga R, González F, Vera F, González F. Discordancia entre el perfil declarado y el observado en coordinadores de procuramiento de órganos en Chile. Libro de resúmenes del VII Congreso Chileno de Trasplante 2019, página 71. [En línea]. Disponible en: http:// congreso2019.sociedaddetrasplante.cl/assets/libro-resumen-congreso-trasplante-2019.pdf p.71.

19. Vera M, Alegría L, Domínguez J, Rebolledo R, Dib M. Donación y trasplante de órganos: análisis y propuestas para avanzar hacia una cultura de donación. Temas de la Agenda Pública, 14 (117), 1-20. Centro de Políticas Públicas UC. [En línea]. Disponible en: http://políticas- 
publicas.uc.cl/publicación/serie-temas-de-la-agenda/ donacion-y-trasplante-de-organos-analisis-y-propuestas-para-avanzar-hacia-una-cultura-de-donacion/

20. Cuidados Intensivos orientados a la donación de órganos. Recomendaciones. Grupo de trabajo SEMICYUC-ONT Septiembre de 2017 [En línea]. Disponible en http://www.ont.es/mailings/CIOD_Recomendaciones\%20SEMICYUCONT_Sseptiembre2017.pdf [Accedido: 24 de noviembre de 2019].

21. Beca JP. Bioética y trasplante de órganos de cadáver.
Contacto Científico. 20 años de Trasplantes de hígado. 2013; 23-26. [En línea]. Disponible en: http:// contactocientifico.alemana.cl/ojs/index.php/cc/article/ view/135.

22. Cluzet O. Trasplante órganos sólidos: bases éticas de su estructuración. En Beca JP, Astete C (eds.). Bioética Clínica. Editorial Mediterráneo, Santiago, Chile 2012; pp 568-81.

23. Manson NC. Normative consent is not consent. Cam $Q$ Healthc Ethic 2013; 22: 33-44. 